\title{
Co-simulation of of Finite Element and Circuit Solvers using Optimized Waveform Relaxation
}

\author{
Jean de Dieu Nshimiyimana, Frédéric Plumier, \\ Patrick Dular, Christophe Geuzaine \\ Dept. of Electrical Engineering and Computer Science \\ University of Liege, ULG \\ Belgium \\ jeandedieu.nshimiyimana@student.ulg.ac.be
}

\author{
Johan Gyselinck \\ Ecole Polytechnique de Bruxelles \\ Université Libre de Bruxelles, ULB \\ Belgium
}

\begin{abstract}
This paper focuses on a comparison of the cosimulation schemes for coupled problems with application to coupled electromagnetic field and power-electronic simulations. A co-simulation framework between a finite element solver and a circuit solver based on the waveform relaxation scheme results in an iterative process whose convergence depends on the kind of transmission coupling conditions (TCC) on the interface between the sub-problems. The convergence of TCC is illustrated on two test cases: a boost converter and a switched reluctance motor drive.

Index terms - co-simulation, optimized waveform relaxation, electrical converters, finite element
\end{abstract}

\section{INTRODUCTION}

Solving field-circuit problems monolithically, i.e., solving both the partial differential equations describing the field problem and the differential-algebraic equations describing the circuit problem can be very challenging. Indeed, on the one hand, if the time scales that need to be resolved in both problems are several orders of magnitude apart (e.g. when coupling circuits with fast switching components with electromechanical models with slower dynamics), using the same discretization for both problems can lead to very high computation times. On the other hand, when existing highly optimized tools exist for solving each problem separately, building a monolithic solver for coupled problem can prove difficult, or downright impossible. For both reasons, decomposing the problem into subproblems that can be solved separately can be advantageous.

Waveform relaxation (WR), also called dynamic iteration [1], is a domain decomposition technique used for solving time-dependent problems, where two or more sub-problems are solved independently for a certain time interval before exchanging data. Potential iterations between sub-problems are performed over the interval until convergence, before proceeding to the next interval. Such methods have been proposed and successfully used for more than 30 years in circuit simulators [2], [3], and more recently in electromagnetic fieldcircuit computations [1].

978-1-4673-8463-6/16/\$31.00 (c) 2016 IEEE
Compared to classical monolithic field-circuit solvers, WR presents several advantages: a co-simulation framework can be set up where the field and the circuit problems are respectively solved by their specialized solvers, and time integration methods can be adapted independently in each sub-problem. The main disadvantage is that several dynamic iterations are usually necessary in order to reach convergence. Keeping the number of iterations small is thus paramount for computational efficiency.

The convergence of WR schemes depends on the mathematical structure of the field and circuit equations. Those, in general, are differential-algebraic equations, which can be analyzed in terms of their index [1]. The choice of the transmission coupling conditions (TCC) between the two subproblems plays a key role: when coupling magnetodynamic finite element (FE) computations with circuit (CIR) simulations, for example, it is known that exchanging the impedance and the voltage between the subproblems leads to faster convergence than simply exchanging the voltage and the current [1].

This paper focuses on the application of WR to electromagnetic field-power-electronic-circuit problems. It provides a comparison of the performance of the different types of TCC on several examples and shows how the classical and optimized waveform relaxation schemes can be interpreted physically in terms of the Thévenin-Norton-equivalents of both sub-problems around a linearization point.

The paper is organized as follows: Section II describes the WR method for coupled problems; Section III illustrates the coupled field-circuit mathematical model; Section IV presents the TCC and Section V-A illustrates the techniques on two applications: a boost converter, and an electrical drive.

\section{WR METHOD FOR COUPLED PROBLEMS}

Let us consider the general form of a coupled problem as a system of implicit ordinary differential equations (ODE) of the form [4]

$$
G\left(x, \frac{d x}{d t}, t\right)=0,
$$


where $x$ is the state-space vector. For example, for electrical circuit networks, the following canonical form can be written:

$G:=\Gamma(x(t), u(t), t) \frac{d x(t)}{d t}-g(x(t), u(t), t)=0, x\left(t_{0}\right)=x_{0}$,

where $\Gamma$ is a matrix obtained by modified nodal analysis [4], $x(t)$ gathers the nodal voltages and the currents in the inductors and the voltage sources, $u(t)$ contains the source voltages and currents and $g$ is assumed to be a sufficiently smooth function. Let us consider a domain decomposition in time where the problem (2) is split into $N$ subproblems, for example based on the time-constant characteristic of each part. Since the matrix $\Gamma$ can be rank deficient, there is possibility that some variables lack derivative connections and are governed by algebraic variables. For $i=1, \ldots, N$ subproblems, the following partitioning follows:

$$
\begin{aligned}
& \Gamma_{i}\left(\bar{x}_{i}, \bar{y}_{i}, \bar{u}_{i}, t\right) \frac{d x_{i}(t)}{d t}=g_{i}\left(\bar{x}_{i}, \bar{y}_{i}, \bar{u}_{i}, t\right), \\
& 0=h_{i}\left(\bar{x}_{i}, \bar{y}_{i}, \bar{u}_{i}, t\right), \\
& \bar{x}_{i}\left(t_{0}\right)=\bar{x}_{i 0} .
\end{aligned}
$$

where

$$
\begin{aligned}
\bar{x}_{i} & =\left[x_{1}, \ldots, x_{i-1}, x_{i}, x_{i+1}, \ldots, x_{N}\right], \\
\bar{y}_{i} & =\left[y_{1}, \ldots, y_{i-1}, y_{i}, y_{i+1}, \ldots, y_{N}\right], \\
\bar{u}_{i} & =\left[u_{1}, \ldots, u_{i-1}, u_{i}, u_{i+1}, \ldots, u_{N}\right] .
\end{aligned}
$$

In this splitting, $\Gamma_{i}, x_{i}, u_{i}$ and $g_{i}$ represent the restriction of the original operators and vectors on the sub-problem $i, y_{i}$ is an interface variable for subsystem $i, g_{i}$ is the restriction of $g$ on subproblem $i$ and $h_{i}$ is the coupling function for the state and algebraic (nonstate) variables with respect to the $\mathrm{i}$-th subproblem. For equation (4) to be solvable, $\frac{\partial h_{i}}{\partial \bar{y}_{i}}$ has to be regular, which means, by the implicit function theorem, that each subsystem $h_{i}\left(\bar{x}_{i}, \bar{y}_{i}, \bar{u}_{i}\right)=0$ should be (locally) uniquely solvable for $\bar{y}_{i}$. If so, system (3) and (4) is called a system of differential algebraic equations (DAEs).

Solving the full problem then involves iterating between the subproblems since a number of variables $(y)$ are shared between them.

Solving (3)-(4) involves first substructuring the simulation time interval $\left[t_{0}, T\right]$ into subintervals $T_{\iota}=\left[t_{\iota}, t_{\iota+1}\right]$ of length $H_{\iota}=t_{\iota+1}-t_{\iota}$ with $\iota=0,1, \ldots M$ the time discretization index and synchronizing points $t_{\iota}$ such that $t_{0}<t_{1} \ldots<t_{M}=T$. Assuming that $\Gamma_{i}^{-1}$ exists, such that $f_{i}:=\Gamma_{i}^{-1} g_{i}$, the GaussSeidel type WR algorithm reads as follows for two subproblems, i.e., $i=1,2$ :

1) Set the WR iteration index $k=0$; and the time discretization index $\iota=0$ and guess the initial waveforms $x_{2}^{0}(t)$ and $y_{2}^{0}(t) ; t \in\left[t_{0}, t_{1}\right]$ with $x_{1}^{0}\left(t_{0}\right)=$ $x_{10}, x_{2}^{0}\left(t_{0}\right)=x_{20}$ and such that

$$
\begin{aligned}
& h_{1}\left(x_{1}^{0}\left(t_{0}\right), x_{2}^{0}\left(t_{0}\right), y_{1}^{0}\left(t_{0}\right), y_{2}^{0}\left(t_{0}\right), u_{1}\left(t_{0}\right), u_{2}\left(t_{0}\right)\right)=0 \\
& h_{2}\left(x_{1}^{0}\left(t_{0}\right), x_{2}^{0}\left(t_{0}\right), y_{1}^{0}\left(t_{0}\right), y_{2}^{0}\left(t_{0}\right), u_{1}\left(t_{0}\right), u_{2}\left(t_{0}\right)\right)=0
\end{aligned}
$$

2) While $k \leq N_{k}$, a) Solve

$$
\begin{aligned}
\frac{d x_{1}^{k+1}}{d t} & =f_{1}\left(x_{1}^{k+1}, x_{2}^{k}, y_{1}^{k+1}, y_{2}^{k}, u_{1}, u_{2}, t\right), \\
0 & =h_{1}\left(x_{1}^{k+1}, x_{2}^{k}, y_{1}^{k+1}, y_{2}^{k}, u_{1}, u_{2}, t\right),
\end{aligned}
$$

b) Solve

$$
\begin{aligned}
\frac{d x_{2}^{k+1}}{d t} & =f_{2}\left(x_{2}^{k+1}, x_{1}^{k+1}, y_{1}^{k+1}, y_{2}^{k+1}, u_{1}, u_{2}, t\right) \\
0 & =h_{2}\left(x_{1}^{k+1}, x_{2}^{k+1}, y_{1}^{k+1}, y_{2}^{k+1}, u_{1}, u_{2}, t\right)
\end{aligned}
$$

c) If $\left\|x_{1}^{k+1}-x_{1}^{k}\right\|+\left\|x_{2}^{k+1}-x_{2}^{k}\right\|>T o l$, set $k=$ $k+1$, update $x_{2}^{k}=x_{2}^{k+1}, y_{2}^{k}=y_{2}^{k+1}$ and go to 2 ); otherwise the WR has converged and go to 3 )

3) If $t_{\iota+1}<T_{N}$, set $x_{1}^{0}\left(t_{\iota+1}\right)=x_{1}^{k}\left(t_{\iota+1}\right), k=0, \iota=\iota+1$ and guess the waveforms $x_{2}^{0}(t)$ and $y_{2}^{0}(t), t \in\left[t_{\iota}, t_{\iota+1}\right]$ and then go to 2).

This Gauss-Seidel scheme is inherently sequential, since subproblem $i$ must be solved before subproblem $j$ if $j>i$. A Jacobi-type WR can be formulated to alleviate this problem:

$$
\begin{aligned}
\frac{d x_{1}^{k+1}}{d t} & =f_{1}\left(x_{1}^{k+1}, x_{2}^{k}, y_{1}^{k+1}, y_{2}^{k}, u_{1}, u_{2}, t\right), \\
0 & =h_{1}\left(x_{1}^{k+1}, x_{2}^{k}, y_{1}^{k+1}, y_{2}^{k}, u_{1}, u_{2}, t\right), x_{1}^{k+1}\left(t_{0}\right)=x_{10}, \\
\frac{d x_{2}^{k+1}}{d t} & =f_{2}\left(x_{1}^{k}, x_{2}^{k+1}, y_{1}^{k}, y_{2}^{k+1}, u_{1}, u_{2}, t\right), \\
0 & =h_{2}\left(x_{1}^{k}, x_{2}^{k+1}, y_{1}^{k}, y_{2}^{k+1}, u_{1}, u_{2}, t\right), x_{2}^{k+1}\left(t_{0}\right)=x_{20} .
\end{aligned}
$$

The time integration of equations (5)-(7) can be carried out by any adequate time integration scheme (low or high-order, adaptive or not), according to its internal structure, time scales and level of stiffness.

\section{COUPled FIEld-CirCuit PRoBlems}

For coupled field-circuit problems, the electromagnetic field is governed by Maxwell's equations [5], [6]. We consider the quasi-static approximation and an $\mathbf{a}-V$ finite element formulation in terms of the vector magnetic and scalar electric potentials [7], discretized using Whitney elements: $\mathbf{a}(\mathbf{x}, t)=$ $\sum_{i} a_{i}(t) \mathbf{s}_{i}(\mathbf{x}), V(\mathbf{x}, t)=\sum_{i} u_{i}(t) \psi_{i}(\mathbf{x})$. The circuits equations are derived using the modified nodal analysis technique, as briefly presented above.

The coupled field-circuit system reads:

$$
\Gamma \frac{d X}{d t}+\Gamma^{\prime} X=\Phi F^{T},
$$

with $X=\left[a, u, i_{L}, i_{V}\right]$ gathers the coefficients of the magnetic and electric potentials as well as the inductor and voltage source currents. Matrices $\Gamma, \Gamma^{\prime}$, $\Phi$ and $F$ are build classically from the finite element stiffness and mass matrices and the circuit modified nodal analysis.

The formation of the weakly coupled sub-problems follows naturally: we assume that $\left\|\left[\bar{\Gamma}, \bar{\Gamma}^{\prime}\right]\right\|=\left\|\bar{\Gamma} \bar{\Gamma}^{\prime}-\bar{\Gamma}^{\prime} \bar{\Gamma}\right\| \leq \varepsilon[8]$, meaning that each of the system

$$
\bar{\Gamma}_{i} \frac{d x_{i}}{d t}=\bar{\Gamma}_{i}^{\prime} x_{i}+\bar{\Gamma}_{i}^{\prime \prime} y_{i}+\bar{\Phi}_{i} F_{i}, x_{i}(0)=x_{i 0}, i=1,2
$$




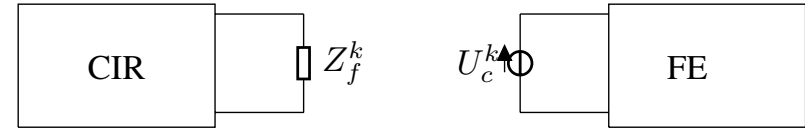

a) VZ-Conditions

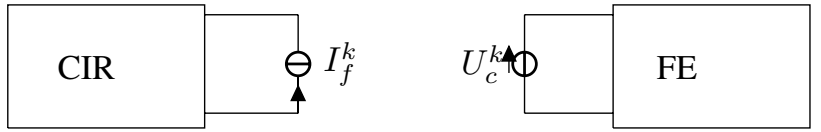

b) VI-Conditions

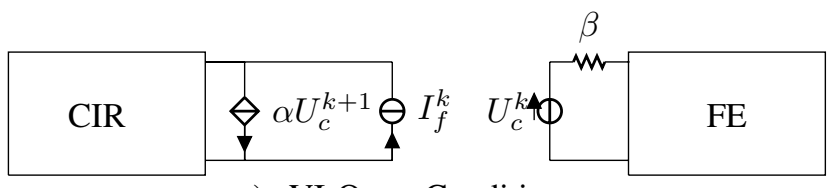

c) VI-Opt $0 .-$ Conditions

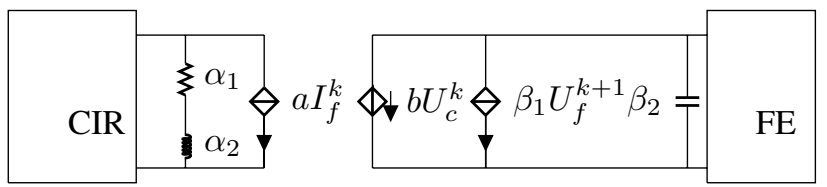

c) VI-Opt 1 -Conditions: a,b are gain constants

Fig. 1: Transmission coupling conditions.

can be solved independently. Notice that this equation follows the general model (2) presented earlier.

\section{TRANSMISSION CONDITIONS}

The convergence of the WR depends on the TCC at the interface where coupled systems are split. We investigate four types of coupling conditions, linked to an underlying equivalent circuit representation of the finite element subsystem, through either a voltage or current source, an impedance or the combination of both. Four TCCs are considered (see Figure $1)$ :

- Voltage-Impedance (VZ),

- Voltage-Current (VI),

- Zero-order Optimized Voltage-Current (O0),

- First-order Optimized Voltage-Current (O1).

\section{A. Voltage-Impedance (VZ) TCC}

In this case the first subproblem is represented by its impedance whereas the second is represented by the voltage terminal. These conditions can be modeled as follows:

$$
y_{1}:=X_{01}^{k+1}=C_{0}^{\prime} x_{2}^{k} ; y_{2}:=X_{02}^{k+1}=C_{0} x_{1}^{k+1}
$$

which correspond to the voltage $U_{c}$ and current $I_{f}$ at the boundary of the circuit and the FE subsystems (see Fig. 1 a.) A WR algorithm is applied on the semi-discretized equations (9). Both systems together with the boundary conditions form the following quasi-linear-DAE [4]:

$$
\begin{aligned}
& \hat{\Gamma}_{1} \frac{d}{d t} x_{1}^{k+1}=\hat{\Gamma}_{1}^{\prime} x_{1}^{k+1}+\Gamma_{1}^{\prime \prime} C_{0}^{\prime} x_{2}^{k}+\Phi_{1} F_{1} ; \\
& \hat{\Gamma}_{2} \frac{d}{d t} x_{2}^{k+1}=\hat{\Gamma}_{2}^{\prime} x_{2}^{k+1}+\Gamma_{2}^{\prime \prime} C_{0} x_{1}^{k+1}+\Phi_{2} F_{2} ;
\end{aligned}
$$

where $C_{0}, C_{0}^{\prime}, \Gamma_{1}^{\prime \prime}, \Gamma_{2}^{\prime \prime}$ are the interface matrices.
1) Convergence Rate: The determination of the convergence rate in the linear case is equivalent to finding the spectral radius of the WR iteration matrix, which can be obtained from algebraic equations by using the Laplace Transform (LT) on the DAEs. Applying the LT to (11) yields:

$$
\begin{aligned}
& s \hat{\Gamma}_{1} \hat{x}_{1}^{k+1}=\hat{\Gamma}_{1}^{\prime} \hat{x}_{1}^{k+1}+\Gamma_{1}^{\prime \prime} C_{0}^{\prime} \hat{x}_{2}^{k}+\Phi_{1} F_{1} ; \\
& s \hat{\Gamma}_{2} \hat{x}_{2}^{k+1}=\hat{\Gamma}_{2}^{\prime} \hat{x}_{2}^{k+1}+\Gamma_{2}^{\prime \prime} C_{0} \hat{x}_{1}^{k+1}+\Phi_{2} F_{2} .
\end{aligned}
$$

We ignore the source terms here since they do not appear in the error term. Using the initial conditions $x_{1}(0)=x_{2}(0)=0$, andreordering terms in (12) leads to

$$
\begin{array}{r}
\hat{x}_{1}^{k+1}=\Pi_{V Z} \hat{x}_{1}^{k-1} \text { with } \Pi_{V Z}=\Pi_{1}^{2}, \text { and } \\
\Pi_{1}=\left(s \hat{\Gamma}_{2}-\hat{\Gamma}_{2}^{\prime}\right)^{-1} \Gamma_{2}^{\prime \prime} C_{0}\left(s \hat{\Gamma}_{1}-\hat{\Gamma}_{1}^{\prime}\right)^{-1} \Gamma_{1}^{\prime \prime} C_{0}^{\prime} ;
\end{array}
$$

and $\Pi_{1}$ is the iteration matrix. The spectral radius of the iteration matrix is found as follows:

$$
\rho_{V Z}=\max \left|\sigma\left(\Pi_{V Z}\right)\right|
$$

where $\sigma\left(\Pi_{V Z}\right)$ is the set of the eigenvalues.

\section{B. Voltage-current (VI) TCC}

This case corresponds to the equivalent circuit shown in Fig. $2 \mathrm{~b})$, and can be represented as follows:

$$
y_{1}:=X_{01}^{k+1}:=C_{0}^{\prime} x_{2}^{k} ; y_{2}:=X_{02}^{k}:=C_{0} x_{1}^{k} ;
$$

The spectral radius can be determined in a similar way as for the VZ case, leading to

$$
\rho_{V I}=\max \left|\sigma\left(\Pi_{V I}\right)\right| ;
$$

where $\Pi_{V I}=\Pi_{1}$. Note that the iteration matrix in the $V Z$ is $\Pi_{1}^{2}$.

\section{Zero-order optimized voltage-current TCC}

In this TCC, the transmission condition (15) is redefined in such a way that there is a matching condition between the subsystems. An equivalent circuit for zero-order optimized WR is presented in Fig. 2c), where the parameters $\alpha$ and $\beta$ are introduced to enhance the WR convergence. These conditions are given as follows:

$$
\begin{aligned}
& X_{01}^{k+1}+\alpha C_{0} x_{1}^{k+1}=C_{0}^{\prime} x_{2}^{k}+\alpha X_{02}^{k} \\
& X_{02}^{k+1}+\beta C_{0}^{\prime} x_{2}^{k+1}=C_{0} x_{1}^{k}+\beta X_{01}^{k} .
\end{aligned}
$$

This kind of transmission conditions are called zero-order transmission conditions [9] since they don't involve any derivative of the variables at the boundary of the subproblems. The computation of the iteration matrix is done like in the preceding case and we find:

$$
\hat{x}_{1}^{k+1}=\Pi_{O 0} \hat{x}_{1}^{k-1}
$$

where

$$
\begin{aligned}
\Pi_{O 0}= & \left(s \hat{\Gamma}_{1}-\hat{\Gamma}_{1}^{\prime}+\alpha \Gamma_{2}^{\prime \prime \prime}\right)^{-1} \Gamma_{1}^{\prime \prime}\left(C_{0}^{\prime}-\alpha \beta C_{0}^{\prime}+\alpha \cdot\right. \\
& \left.\Gamma_{2}^{\prime \prime-1}\left(s \hat{\Gamma}_{2}-\hat{\Gamma}_{2}^{\prime}+\beta \Gamma_{1}^{\prime \prime \prime}\right)\right)\left(s \hat{\Gamma}_{2}-\hat{\Gamma}_{2}^{\prime}+\beta \Gamma_{1}^{\prime \prime \prime}\right)^{-1} \Gamma_{2}^{\prime \prime}\left(C_{0}-\right. \\
& \left.\alpha \beta C_{0}+\beta \Gamma_{1}^{\prime \prime-1}\left(s \hat{\Gamma}_{1}-\hat{\Gamma}_{1}^{\prime}+\alpha \Gamma_{2}^{\prime \prime \prime}\right)\right)
\end{aligned}
$$




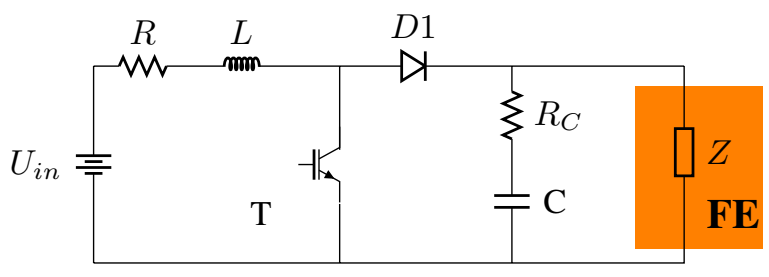

Fig. 2: DC-DC boost-converter model.

TABLE I: Geometric and material data for the FE load.

\begin{tabular}{|c|c|c|c|c|c|}
\hline & width & length $(\mathrm{m})$ & depth $(\mathrm{m})$ & $\sigma\left(\frac{S}{m}\right)$ & $\mu\left(\frac{H}{m}\right)$ \\
\hline coil & 0.08 & 0.02 & 0.05 & $5.77 \mathrm{e} 7$ & $\mu_{0}$ \\
core & 0.10 & 0.08 & 0.0508 & $0.5 \mathrm{e} 6$ & 2500 \\
\hline
\end{tabular}

The convergence rate is found by the minimization of spectral radius of the iteration matrix as follows,

$$
\rho_{O 0}=\min _{\alpha<0, \beta>0} \max _{0<\omega_{\min }<\omega<\omega_{\max }}\left|\sigma\left(\Pi_{O 0}\right)\right|,
$$

where $\omega$ is the circular frequency and is such that the LT argument $s=i \omega$.

The first condition of the transmission conditions (17) can be viewed as a Thévenin equivalent law where $\beta$ is the impedance; the second one can be viewed as the Norton equivalent law where $\alpha$ is the conductance.

\section{First-order optimized voltage-current TCC}

The optimized first-order transmission condition consists in introducing not only the boundary variables but also their derivatives. These conditions thus take the following form:

$$
\begin{aligned}
& \alpha_{1} X_{01}^{k+1}+\alpha_{2} \frac{d X_{01}^{k+1}}{d t}+C_{0} x_{1}^{k+1}=\alpha_{1} C_{0}^{\prime} x_{2}^{k}+\alpha_{2} \frac{C_{0}^{\prime} d x_{2}^{k}}{d t}+X_{02}^{k}, \\
& \beta_{1} X_{02}^{k+1}+\beta_{2} \frac{d X_{02}^{k+1}}{d t}+C_{0}^{\prime} x_{2}^{k+1}=\beta_{1} C_{0} x_{1}^{k}+\beta_{2} \frac{C_{0} d x_{1}^{k}}{d t}+X_{01}^{k} .
\end{aligned}
$$

The computation of the iteration matrix leads to:

$$
\hat{x}_{1}^{k+1}=\Pi_{O 1} \hat{x}_{1}^{k-1} ;
$$

where

$$
\begin{gathered}
\Pi_{O 1}=\left(s \hat{\Gamma}_{1}-\hat{\Gamma}_{1}^{\prime}+\alpha_{1} \hat{\Gamma}_{2}^{\prime \prime \prime}\right)^{-1} \hat{\Gamma}_{1}\left(\left(\alpha_{1}+\alpha_{2} s-\left(\beta_{1}+\beta_{2} s\right)^{-1}\right)\right) \\
C_{0} \Gamma_{1}^{\prime \prime}+\left(\beta_{1}+\beta_{2} s\right)^{-1} \hat{\Gamma}_{2}^{\prime \prime-1}\left(s \hat{\Gamma}_{2}-\hat{\Gamma}_{2}^{\prime}\right. \\
\left.\left.+\beta_{1} \hat{\Gamma}_{1}^{\prime \prime}\right)\right)\left(s \hat{\Gamma}_{2}-\hat{\Gamma}_{2}^{\prime}+\beta \Gamma_{1}^{\prime \prime \prime}\right)^{-1} \Gamma_{2}^{\prime \prime}\left(C_{0}-\right. \\
\left.\quad \alpha \beta C_{0}+\beta \Gamma_{1}^{\prime \prime-1}\left(s \hat{\Gamma}_{1}-\hat{\Gamma}_{1}^{\prime}+\alpha \Gamma_{2}^{\prime \prime \prime}\right)\right)
\end{gathered}
$$

Again, the convergence rate is found by the minimization of the spectral radius of the iteration matrix:

$$
\rho_{O 1}=\min _{\alpha_{1}>0, \alpha_{2}>0, \beta_{1}>0, \beta_{2}>0} \max _{0<\omega_{\min }<\omega<\omega_{\max }}\left|\sigma\left(\Pi_{O 1}\right)\right| .
$$

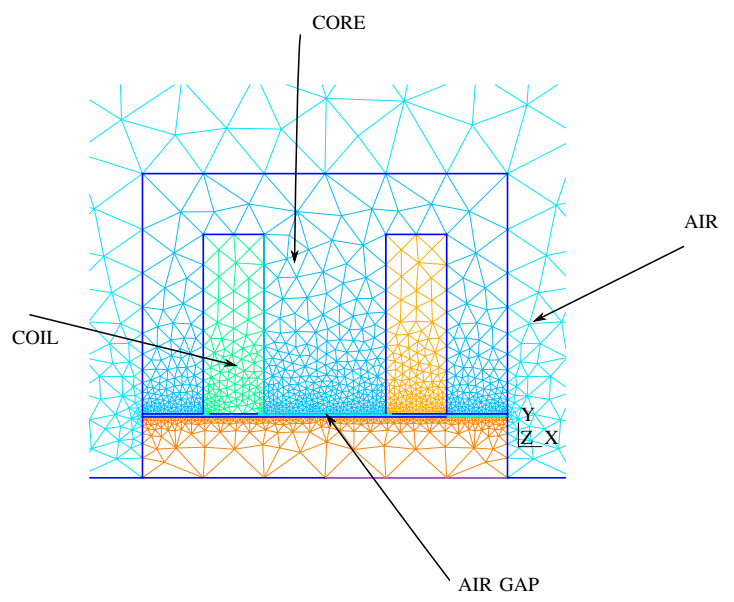

Fig. 3: FE Inductor mesh geometry.

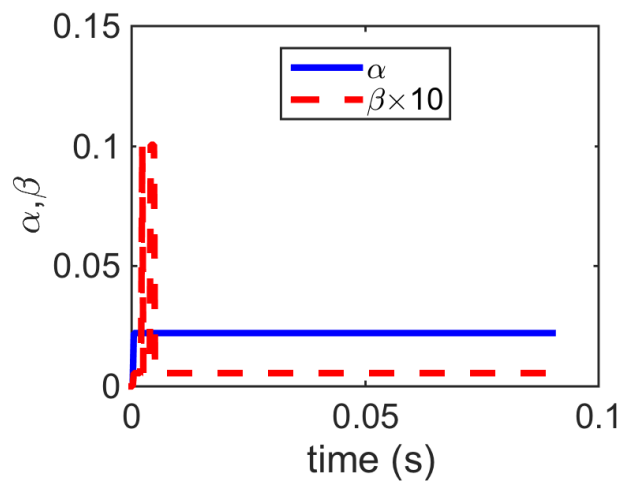

Fig. 4: Optimized parameters for the Boost example.

\section{ApPliCATIONS}

\section{A. DC-DC Boost Converter}

Power electronics converters are a prime candidate for illustrating the WR method for coupled electromagnetic fieldcircuit problems. Indeed, while such converters can be analyzed purely with lumped elements, the high simulation accuracy required by modern applications can make it necessary to simulate in detail the electromagnetic field distribution in some of the power converters magnetic elements, especially when saturation can occur. The cohabitation of the magnetic components with the fast switching control systems makes a monolithic approach unpractical: WR with adapted time steps in each of the two subsystems is thus a natural choice.

As a simple test case, we focus on a DC-DC boost-converter (see Fig. 2) with an RL load analyzed with FE. This example allows to test the convergence behavior linked to the different TCCs. When the load is more resistive, the pulse width modulation (PWM) of the transistor gate is not felt by the magnetic inductor. But when the load becomes more inductive, the on-off states of the transistor are detected (see Fig. 6) and this becomes critical to the behavior of the TCC. 


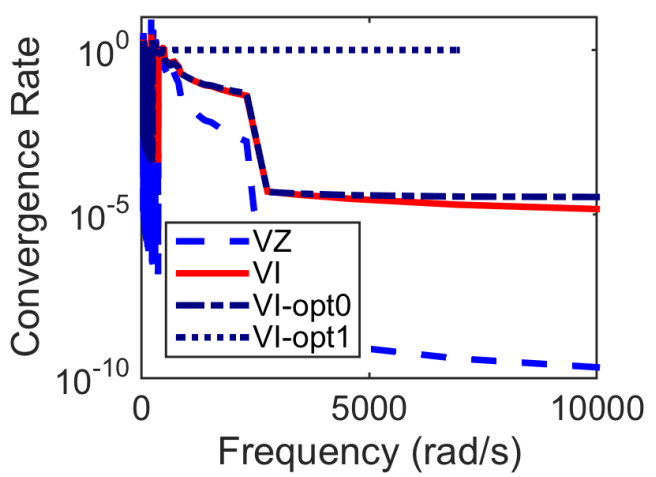

Fig. 5: Comparison of convergence rates for the Boost example.

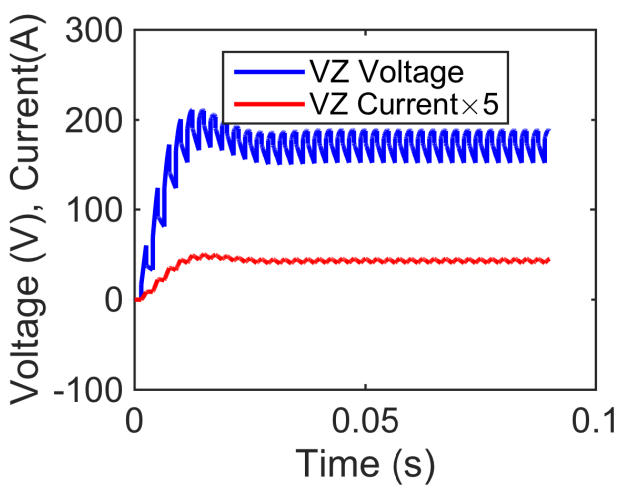

Fig. 6: Interface variables for boost model: mostly resistive load

The boost-converter design parameters are chosen as follows:

$$
\begin{aligned}
& C \geq \frac{I_{O}(\max ) \times\left(1-\sqrt{\frac{2 \times L}{R_{C} \times T_{s}}}\right)}{f_{s} \times \Delta V_{O}}, L \geq \frac{V_{O}(\max ) \times T_{s}}{16 \times I_{O(\text { crit })}}, \\
& D=1-\frac{V_{\text {in }}}{V_{\text {out }}},
\end{aligned}
$$

where $f_{s}, T_{s}, I_{o(\max )}, U_{\text {in }}, U_{\text {out }}, \Delta V_{0}, D$ are respectively the switching frequency, the period, the output maximum current, the input voltage, the maximum output voltage, the output voltage ripple and the duty cycle. More details can be found in [10].

Fig.3 shows the geometry and the mesh for the FE load [11]. The geometry properties and the material constant parameters are presented in the Table I. Here $\mu_{0}=4 \pi e-7$ is the permeability of the air.

Fig. 4 presents the zero-order optimized parameters time evolution for the linearized Boost example presented in section V-A. Fig. 5 compares the spectral radius $\rho_{V Z}, \rho_{V I}, \rho_{0 O}$, and $\rho_{01}$, versus the frequency $\omega$. The firstorder optimized parameters are found as $\alpha_{1} \in[3,3.5], \alpha_{2} \in$ $[4,4.5], \beta_{1}=1 e-3, \beta_{2}=0$.

Fig. 6 and 7 show the interface voltage and current waveforms for a more resistive or inductive load, respectively. Fig. 8 shows the dynamic waveform relative error (for the first time window) for all four TCC in the latter, more challenging

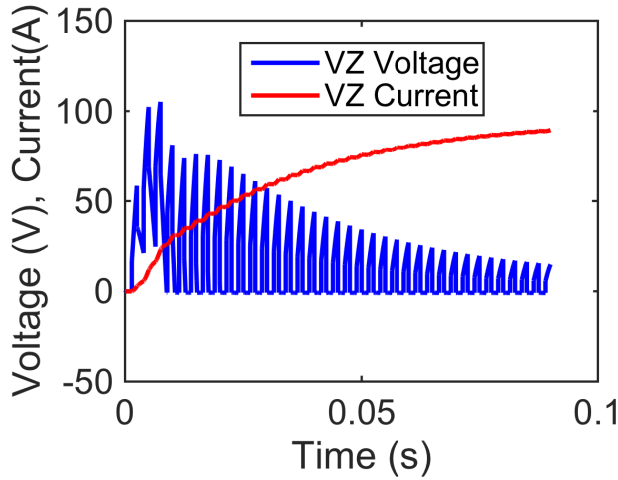

Fig. 7: Interface variables for boost model: mostly inductive load

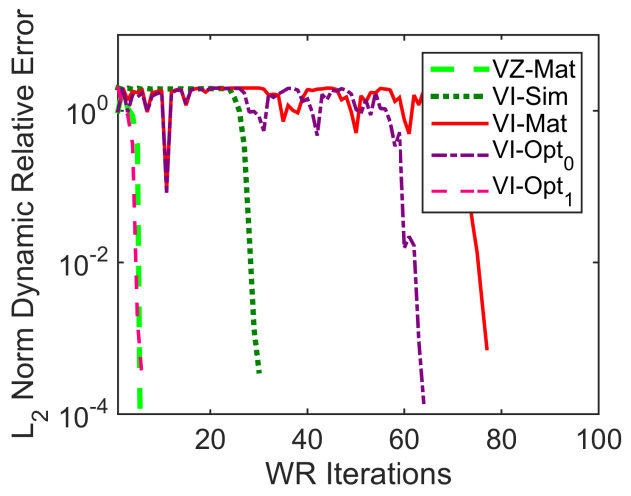

Fig. 8: Dynamic relative error on the voltage and current at the interface for the boost model. ("Mat" denotes fixed step circuit integration; "Sim" denotes adaptive integration performed with Simulink Simpower Systems.)

case. The impedance-voltage coupling transmission condition and the optimized first-order TCC clearly outperform both the Voltage-Current and the optimized zero-order Voltage-current TCC.

\section{B. Switched Reluctance Motor (SRM)}

WR is now applied on a three phase SRM, fed by a coupled boost-inverter drive (Fig. 9). Only the VZ TCC is considered, i.e., each of the SRM phases is represented by its impedance in the circuit model and the circuit is represented by the phase voltage terminal from the circuit boundary. The integration of the stator phase equivalent circuit model is done by integrating directly the flux linkage [12] obtained through the FE model [11]. The current in the excited phase is found as

$$
I_{f}^{k+1}=\frac{\int_{0}^{T}\left(V_{L}^{k+1}-R_{s} I_{f}^{k+1}\right)+L_{s}^{k}(0) I_{f}^{k}(0)}{L_{s}^{k}} .
$$

Fig. 10 shows the voltage and current at the interface between the machine and the drive. Fig. 11 presents dynamic relative error on the current for the first time window. On this realistic example, convergence is attained after 4 iterations. 


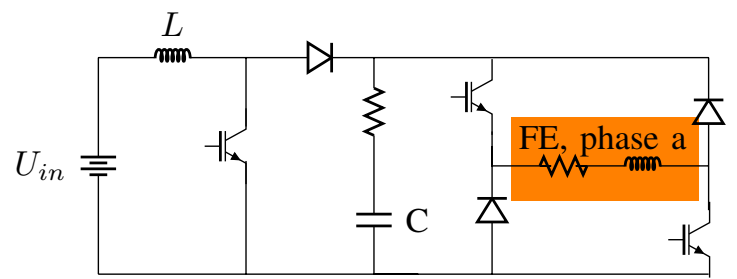

Fig. 9: Boost-converter and asymetric H-bridge inverter.

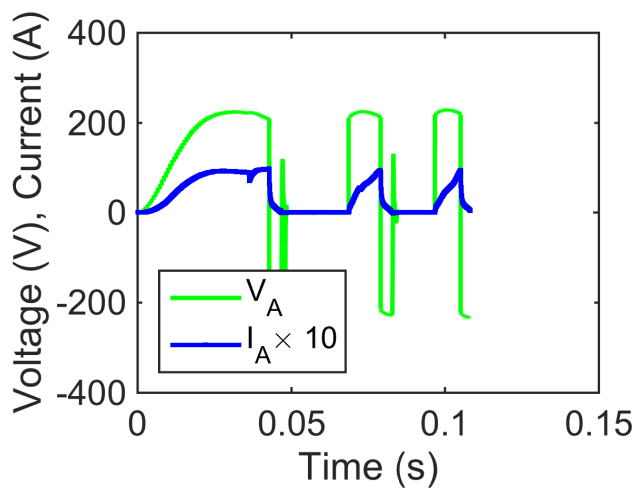

Fig. 10: Phase A voltage for the SRM example.

\section{CONCLUSION}

This paper presented and analyzed four different types of transmission coupling conditions for the simulation of coupled electromagnetic-field-circuit problems using Waveform Relaxation methods. Voltage-impedance and optimized first order conditions lead to fast convergence of the iterative process, and have been successfully applied to two practical applications: a boost power electrics converter and a switched reluctance motor.

\section{ACKNOWLEDGEMENTS}

This work was supported in part by Wallonie-Bruxelles International (WBI), the Belgian Science Policy (IAP P7/02) and the Walloon Region of Belgium (WBGreen Grant FEDO).

\section{REFERENCES}

[1] S. Schöps, H. De Gersem, and A. Bartel, "A cosimulation framework for multirate time integration of field/circuit coupled problems," Magnetics, IEEE Transactions on, vol. 46, no. 8, pp. 3233-3236, 2010.

[2] E. Lelarasmee, A. E. Ruehli, and A. Sangiovanni-Vincentelli, "The waveform relaxation method for time-domain analysis of large scale integrated circuits," Computer-Aided Design of Integrated Circuits and Systems, IEEE Transactions on, vol. 1, pp. 131-145, July 1982.

[3] G. Martin Jacob and R. Albert E., "Optimized waveform relaxation methods for rc type circuits," IEEE Transactions on Circuits and Systems. 1, vol. 51, no. 4, pp. 755-768, 2003.

[4] P. J. Rabier, Theoretical and Numerical Analysis of Differential Algebraic Equations. PA 15260,USA,2002: Department of Mathematicas, University of Pittsburgh, 2002.

[5] R. Dautray and J. Lions, Analyse mathématique et calcul numérique pour les sciences et les techniques. No. vol. 1 in Analyse mathématique et calcul numérique / Robert Dautray, Jacques-Louis Lions, Masson, 1987.

[6] J.-M. Jin, The finite element method in electromagnetics. John Wiley \& Sons, Inc., 2002.

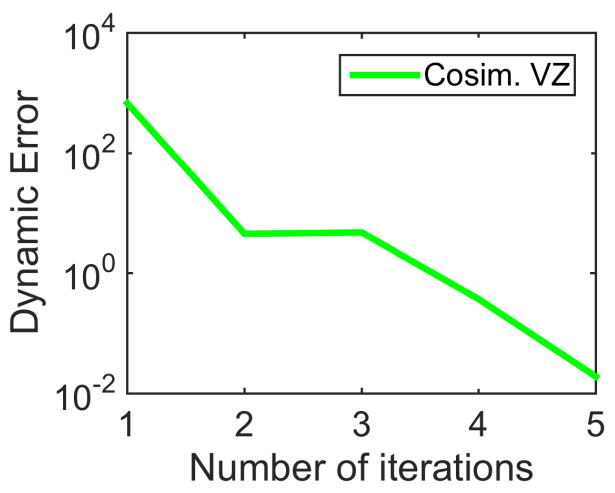

Fig. 11: Dynamic dynamic relative error for the SRM example.

[7] P. Dular, F. Henrotte, and W. Legros, "A general and natural method to define circuit relations associated with magnetic vector potential formulations," Magnetics, IEEE Transactions on, vol. 35, pp. 16301633, May 1999.

[8] J. Geiser, Coupled Systems: Theory, Models, and Application in Engineering. (CRC Press. A chapman \& Hall/CRC Numerical Analysis and Scientific Computing Series), 2014.

[9] M. AI-Khaleel, Optimized Waveform Relaxation Methods for RC Type Circuits. McGill University, Montreal: Department of Mathematics and Statistics, McGill University, Montreal, 2002.

[10] S. S. Ang, Power-Switching Converters. Marcel Dekker, Inc., 270 Madison Avenue, New york 10016: United State of America, 1995.

[11] "Onelab project." online: http://onelab.info.wiki/machines.

[12] "Modeling time-varying storage components in pspice." online: http: //user.unob.cz/biolek. 